\title{
Nuclear Effective Field Theories: Reverberations of the early days
}

\author{
U. van Kolck \\ Université Paris-Saclay, CNRS/IN2P3, IJCLab, \\ 91405 Orsay, France \\ and \\ Department of Physics, University of Arizona, \\ Tucson, AZ 85721, USA
}

July 27, 2021

\begin{abstract}
Effective field theories are recognized nowadays as the framework to describe low-energy nuclear structure and reactions consistently with the underlying theory of the strong interactions, QCD. It was not always so. As we celebrate the 30 years of the first publications, I collect here some memories from those early days. I also discuss some of the key issues that were raised back then and, despite all progress, are still with us.
\end{abstract}

Dedicated to the memory of Steven Weinberg, whose ideas will not die, wherever they may lead. 


\section{An Anecdote}

As I am walking down the hall on the 9th floor of the RLM building at the University of Texas at Austin sometime in 1990, a visibly agitated Steve Weinberg comes out of his office, makes a beeline for me, and says (approximately) "Bira, I just got off the phone with Gerry Brown. I called him with some questions because he is one of the statesmen in nuclear physics. But all he talks about is fitting data and the rho meson! It only makes sense to worry about fitting data once you have a theory that is internally consistent. You should not just add ingredients to fit data!" Steve had expressed this point of view many times before 1. In fact, this is one of the tenets of a product of Steve's genius, effective field theories (EFTs) [2]: you consider the most general dynamics consistent with the lowenergy degrees of freedom and symmetries, you order these interactions according to the powers of the ratio of relevant scales that you expect they will contribute to observables ("power counting"), and you calculate these observables order by order in the expansion. If you are lucky - and boy, have we been lucky in nuclear physics! - it does not work as well as you anticipated, and you learn something. The context of Steve's phone call was our early struggles [3, 4, 5, 6] to formulate an EFT for nuclear physics, which at the time we referred to as "Chiral Lagrangians" and later as "Chiral EFT" because of the prominent role played by the light pions and chiral symmetry. Steve's point was that there should be no particular role for an explicit rho meson in Chiral EFT: the rho is not a low-energy degree of freedom since it is unstable with a mass on the order of the EFT's breakdown scale (and of the masses of everything else that is strong interacting).

In early 1994, now visiting Austin after having finished my PhD, I learn the meaning of the word "entranced" when, out of the blue, I get an email from Gerry (via his secretary) about my proposal with Silas Beane for an EFT with quarks and approximate conformal symmetry [7]. Gerry's interest was piqued by a connection to his influential paper [8] with his close collaborator Mannque Rho, who had been the first to react positively in print [9] to the nuclear EFT idea. Unknown to Mannque, Silas and I did most of the work for our paper while traveling to Roma, Lago di Garda, and Trento, where Mannque organized in September 1993 one of the first ECT* workshops, with chiral symmetry as the central topic. Gerry invites me for an immediate visit to Stony Brook, where I find an exciting atmosphere, much as in Steve's group. For lunch just before my talk, the whole group congregates around a long table in a small library-type room. After a few bites of food and physics, Gerry suddenly gets up and says loudly to me something like "So, young man, you don't know this, but once your advisor screamed at me on the phone, and now I'm gonna scream at you!" Before I have a chance to intervene, Gerry launches onto a long sermon articulating why our lack of faith on an explicit rho means eternal damnation. He tops this by alluding to the unreliability of particle physicists doing nuclear physics

\footnotetext{
${ }^{1}$ For example, "I do not believe that scientific progress is always best advanced by keeping an altogether open mind. It is often necessary to forget one's doubts and to follow the consequences of one's assumptions wherever they may lead - the great thing is not to be free of theoretical prejudices, but to have the right theoretical prejudices. And always, the test of any theoretical preconception is in where it leads." [1]
} 
— echoing a statement in an earlier paper of his 2 — when introducing my talk in the same room. Fortunately I had already planned to start with quotes from this [10] and another prescient article [11], where Gerry pointed out the role of chiral symmetry in suppressing two-pion-exchange forces and pion-exchange currents. In the same papers, he urged the implementation of these ideas, which was exactly what I was giving a talk about. I must have made a reasonable case - or maybe it was just that I did not give up soon afterwards - and for the rest of his life Gerry showed me nothing but support. And, with time, I understood that Gerry's point was not only about data fitting — it also bore on the renormalization, and thus on the consistency, of the EFT.

This story is not a fable despite its undertones of Jungian synchronicity. It presaged much of the cultural rift that has characterized the development of nuclear EFTs in the 30 years that followed: a tension between effective-field theorists searching for a consistent formulation and other nuclear theorists striving to fit data to ever greater precision, oftentimes at the expense of EFT tenets. For the last 20 years there has been an imbalance towards the second approach [12, 13], in large extent because it is easier to implement in the new, powerful "ab initio" methods for the solution of the Schrödinger equation (and its few-body variants) [14.

I will take here my usual Grillo Parlante (aka Jiminy Cricket) role and look back at the early days from the perspective of the consistency issues that worried us then and are still around today. These problems have led [15] not only to a better understanding of power counting in Chiral EFT, but also to the development of two successful lowerenergy EFTs, "Pionless EFT" (where pions are "integrated out") and "Halo/Cluster EFT" (where additionally tight nucleon clusters are "integrated in"). After recollecting memories of the very early days (Sec. 2), I remind the reader of some salient implications of EFT to nuclear physics (Sec. 3). I discuss them in more detail, starting from their roots, in the following sections: the justification of power counting from renormalization and naturalness (Sec. 4); the importance of few-body forces (Sec. 5); consequences for reactions with external probes (Sec. 6); and the role of the Delta isobar (Sec. 7). I then conclude (Sec. 8).

\section{Glory Days}

In the late '80s and early '90s, Weinberg's Theory Group was a special place, and I am not speaking (only) out of nostalgia for the days of youth. It was not for the mere presence of one of the best theorists alive. (I have seen theory demigods waiting nervously at the door of Zeus's office...) It was neither just for the company of other superb theorists - at various times, Philip Candelas, Willy Fischler, Vadim Kaplunovsky, and Joe Polchinski - or exceptional graduate students - in my year and the next, the likes of Silas Beane, Per Berklund, Rafael López-Mobilia, Djordje Minic, and Scott Thomas. It was that Steve

\footnotetext{
2 "Occasionally, these days, particle physicists (...) dive into the meson soup, but come up as soon as they are out of breath, brandishing yet another half-boiled manuscript, running quickly off into other realms where there is much less data to embarrass them." [10]
} 
made sure that there was a culture of open discussion about anything. Rarely, if ever, one of the weekly Monday brown-bag lunch talks or Tuesday afternoon seminars would go by without questions or assessment from Steve. Everything one said to the great man was analyzed in a deep, logical fashion: does it fit with the chain of arguments that starts with quantum field theory as the consistent combination of quantum mechanics and relativity? Everybody, even the string theorists, spoke EFT, and the ethos was that any theoretical problem could, should be addressed.

Steve himself was working on a remarkable set of subjects: string theory, cosmological constant, tests of quantum mechanics, CP violation, restoration of chiral symmetry, constituent quarks, and so on. It came as a surprise to no one, then, when he decided to tackle nuclear forces from the perspective of EFT. Steve's first paper [3] laid the ground for the construction of the nuclear potential and discussed its qualitative properties. Assuming interactions to have strengths ("low-energy constants" or LECs) given by naive dimensional analysis (NDA) [16, 17], scattering amplitudes for processes with at most one nucleon and momenta comparable to the pion mass, $Q \sim m_{\pi}$, are perturbative in a $Q / M_{\mathrm{QCD}}$ expansion, where $M_{\mathrm{QCD}} \sim 1 \mathrm{GeV}$ is the characteristic nonperturbative QCD scale [2]. This sector of Chiral EFT is known as Chiral Perturbation Theory (ChPT) [18]. For two or more nucleons, Steve identified the possible origin of the breakdown in perturbation theory that is responsible for nuclear bound states and resonances: an $m_{N} / Q$ enhancement in "reducible" diagrams whose intermediate states contain only nucleons of mass $m_{N}=\mathcal{O}\left(M_{\mathrm{QCD}}\right)$. He defined the potential as the sum of "irreducible" diagrams, which do not contain this enhancement. Assuming NDA to apply also to LECs of operators involving four or more nucleon fields, he wrote down the form of the leading twoand three-nucleon potentials, leaving higher-nucleon forces to the future.

Steve remembers [19] having the insight of applying ChPT ideas to nuclear physics in 1990 while teaching his two-year course on quantum field theory - which eventually gave rise to his famous books [20, 21, 22]. I rarely missed a class and wish I could recall the moment. I never regarded Steve as a particularly dynamic lecturer; what made his lectures so extremely compelling was the clarity of thought. Each argument was his own and reconstructed - for himself as much as the students - in real time as he spoke. It was common that he paused with a new insight or came back in the next lecture with a better way of expressing an idea.

What I do recall is that Steve soon gave a brown-bag talk to sharpen his thoughts about nuclear forces and invite feedback. I paid particular attention because for my qualifier I worked on a little problem that Steve had suggested earlier: an estimate of axion production in nucleon collisions. I was mesmerized by the work of David Kaplan and others on an EFT for axions [23, 24, 25, but had to deal also with schematic models for pion production. It was an ugly match, but sufficient to pass the exam with a happy Steve. Steve then suggested that I looked into EFTs for the electroweak symmetrybreaking sector, with an eye on the treasures promised by the then-viable SSC. It was soon clear to me that, if I wanted to learn about EFTs in depth, I better study them where data already existed to confront the outcome of power counting. As Gerry would have expected, nuclear forces seemed to me just a good warm-up problem. 
In his brown-bag talk, Steve echoed a remark from his paper about the need to go beyond leading order (LO). The leading potential had some of the ingredients nuclear physicists had assigned to the two-nucleon interaction - one-pion exchange (OPE) and a "hard core", represented by one contact interaction in each $S$ wave. Mannque's paper [9], recommended to me by Steve, brought up another qualitative success of the nuclear EFT idea: since the leading contact interactions had no derivatives, exchange currents were expected to be dominated by pion exchange, in agreement with phenomenology. Still, the leading interactions lacked many features thought to be important or even crucial, for example a spin-orbit force, medium-range attraction, and short-range tensor repulsion. It was thus not clear whether this approach provided a viable foundation for nuclear forces.

Going further looked daunting, and I only jumped in after a discussion with Carlos Ordóñez at the local coffee shop sometime that year. Carlos, then a postdoc wishing to diversify from string theory, provided the needed reassurance about the importance of the project. His research experience, shared cheerfully, was a crucial guide as we navigated new waters. At the same time that we started working on corrections, Steve was writing his second nuclear-force paper [4], which gives a fuller account of, and improves on, the arguments sketched in the first paper. Its main novelty was a power-counting penalty for the loss of connected pieces, which suppresses many-body forces and makes the LO potential purely two-body.

We quickly found with Steve (Sec. 6 of Ref. 4) that, under NDA, parity invariance ensures that next-to-leading-order (NLO) corrections vanish. This was unfortunate as it meant we had to go to at least one order higher $\left(\mathrm{N}^{2} \mathrm{LO}\right)$, which was painful for a couple of reasons. First, we had started before heavy baryon fields were introduced [26] to simplify the counting of derivatives. Second, we had to calculate two-pion exchange (TPE) without double counting iterated OPE. At the time we were using old-fashioned perturbation theory built from the Hamiltonian, which allows for a clean separation of iterated OPE but requires the evaluation of several noncovariant diagrams for each Feynman diagram. On the up side, the $\mathrm{N}^{3} \mathrm{LO}$ two-nucleon potential was easy since no new contact interactions enter and no iterated OPE needs to be subtracted. The isospin-symmetric potential we derived [5] contained the first TPE force consistent with the requirements of chiral symmetry (no large "Z diagrams"!). Already at that point we stressed the role of the (Deltaless) $\mathrm{N}^{3} \mathrm{LO}$ two-nucleon potential in providing the intermediate-range attraction usually thought to be relatively strong and due to the sigma meson and/or correlated pion exchange. Our potential contained all the ingredients of known phenomenological potentials in the form of seven additional two-nucleon contact interactions, as well as short-range components in the three-nucleon potential.

Steve was disappointed that the corrections to the two-nucleon potential were so complex, and decided in his third paper [6] to emphasize three-body forces, be them among nucleons only or involving also pions. He argued that the leading three-nucleon force actually canceled against corrections in the two-nucleon force and focused on pion-deuteron scattering. He suggested a "hybrid" approach where the reaction "kernel" — the externalprobe analog to the potential - is derived from the chiral Lagrangian and sandwiched between wavefunctions obtained from a (mostly two-nucleon) potential taken from else- 
where. After another of Steve's brown-bag talks in the spring of 1992, Ricardo Mastroleo, a visiting professor from Brazil, and I helped Steve with the evaluation of pion-deuteron integrals.

Now, contrary to Steve, I thought the $\mathrm{N}^{3} \mathrm{LO}$ potential was promising. In addition to being accompanied by a prescribed three-nucleon force, the two-nucleon potential was not more complicated than most "realistic" phenomenological potentials. I expected that this would be sufficient to spur further work and I could move on to other things. Fortunately at UT there was an extraordinary nuclear theorist, Lanny Ray, who convinced me that if I wanted to convert others to the gospel, I had to walk the line and fit two-nucleon data. I got similar encouragement later from, among others: Dave Ernst, when Carlos and I visited Texas A\&M University circa 1992; Johan de Swart, Thom Rijken, Rob Timmermans, and the rest of the Nijmegen group, when they welcomed me as family in the summer of '92; and Karl Holinde, when he visited Seattle a couple of years before his untimely passing. To our $\mathrm{N}^{3} \mathrm{LO}$ potential from Ref. [5], Carlos and I added TPE with intermediate Delta isobars, and Lanny adapted the methods he had developed for a phenomenological two-nucleon potential with Delta and Roper [27]. In my PhD thesis [28], I reported preliminary results of a fit to the SAID phase shifts (based on Ref. [29]) at one cutoff.

It became obvious when I ventured away from Austin that Lanny had a fine perception of the nuclear community: it took good notice of the approach but was, with a few notable exceptions, skeptical that it would succeed. Starting with my postdoc interview talks, I was told in no uncertain terms by many people (besides Gerry) that our replacement of heavier-meson exchange by contact interactions had no chance to work, even for the deuteron! I was fortunate to find shelter in the Nuclear Theory Group at the University of Washington. Under Jerry Miller's leadership, the group fostered new ideas and encouraged its junior members to work independently if they chose to - an environment where I could polish, enlarge, and publish parts of my thesis, as well as tackle new challenges. Despite his boundless creativity and stamina, Jerry knew how to strike the perfect balance between stimulation and expectation.

The attitude in the community started to change only after we published the fit to twonucleon phase shifts and deuteron properties. The first publication [30] was essentially the corresponding chapter in my thesis. The second paper 31] contained the full details about the potential and the solution of the Schrödinger equation. We also presented fits to the then-new Nijmegen partial-wave analysis [32] for three different cutoff values. As we emphasized, we had no hope of matching fitting standards of the day, which called for a $\chi^{2} /$ dof $\simeq 1$ up to the pion production threshold. The "realistic" potentials that achieved this were teleological: they added the ingredients needed to reach this objective. In contrast, our point was that a decent fit follows from the theoretical prejudices of EFT. Simplicity was not in the form of the cake, but in its recipe.

One of the first to realize the promise of this approach was Jim Friar, who visited Austin in 1991 or 1992 and immediately became a vocal supporter. In fact, he had anticipated many of the EFT power-counting arguments [33, 34. During his visit he taught us much about nuclear issues not usually stated explicitly in papers. Jim's passion for physics 
meant that he would walk the line with me. I had not met a senior theorist with Jim's dedication to, and ability for, long analytical calculations. I frequently found myself trying to catch up to him. With various collaborators in the following years, we elaborated on two topics that I had only sketched in my thesis: few-nucleon forces and isospin violation.

Jim explained to us that it was well known - at least to those who knew it well... that energy dependence in the two-body potential affected three-body forces. In Chiral EFT, this translates into a cancellation of the corrections to the LO two-nucleon potential that are linear in the energy against both the leading three-nucleon and double-pair diagrams. The cancellation only went through if Steve's three-body force contained a small error. Steve's reaction when I told him was characteristic of his supreme intellectual honesty: he just accepted it with a modest comment. In fact, despite the enormous achievement gap between us he never ever used an argument based on authority, which of course contributed a great deal to my growth as a theorist. The consequence of the cancellation is that the dominant three-nucleon force would come at $\mathrm{N}^{2} \mathrm{LO}$ from the Delta, followed by the $\mathrm{N}^{3} \mathrm{LO}$ force from interactions in the NLO Lagrangian [35]. Among those, TPE is determined by chiral symmetry with a form that had not been properly accounted for before [36]. The shorter-range components, in turn, were shown not to be negligible [37.

Jim was also very interested in isospin violation. In my thesis I emphasized that isospin violation is an accidental symmetry [38, namely an approximate symmetry of the EFT at LO that it is not a symmetry of the underlying theory, here QCD. In my first paper with Jim and Terry Goldman [39], we essentially classified isospin-violating nuclear forces and discussed the most salient interactions. For many years afterwards, we derived various components of the isospin-breaking two- and three-nucleon potentials [40, 41, 42, 43, 44], which complement the isospin-symmetric potentials of Refs. [5, 30, 31].

A final aspect of the early days were reactions. One late Friday afternoon while shooting the breeze with other UT graduate students I had the epiphany that EFT provides the basis for not only nuclear forces but the whole "traditional" nuclear physics. Silas joined enthusiastically. He reminds me that I gave him a draft of Steve's third paper, where he found a factor-of-2 error that Steve had apparently already caught. As I heard from Jim and Justus Koch, who opened the doors of NIKHEF and his home to me in the summer of '92, there was enormous interest in the theoretical interpretation of the new threshold data from Mainz and Saskatoon on neutral-pion photoproduction at threshold - in particular, the novel predictions from ChPT at loop level [18] in disagreement with tree-level phenomenology. For the same reason that loops are relatively important in the single-nucleon sector - the vanishing of LO - threshold neutral-pion photoproduction on the deuteron should be sensitive, Silas and I reasoned, to two-nucleon effects. Silas enlisted a younger UT student, Chang-Yong Lee, in an $\mathrm{N}^{2} \mathrm{LO}$ calculation. Despite the encouragement from Mannque, Ulf Meißner, and especially the late, larger-than-life Aron Bernstein, the project evolved slowly and was published [45] only after Silas and I had left Austin. Still, it served as the basis to go one order further a couple of years later [46]. At this order the proton amplitude agreed well with data, and Silas's insight was that the ChPT amplitude for the neutron could be tested indirectly. An early success 
of nuclear EFT was the confirmation of our prediction (within error bars from the EFT truncation) by a measurement at Saskatoon soon afterwards [47. Encouraged by this success we carried out later with Manuel Malheiro, Judith McGovern and Daniel Phillips a program for the extraction of neutron polarizabilities from Compton scattering on the deuteron [48, 49, 50], which has eventually met with similar success [51]. (See also Daniel's contribution to this volume [52].)

In the meantime, Mannque had assembled a team with his Seoul collaborator Dong-Pil Min and his exceptional student, Tae-Sun Park. Perhaps influenced by Steve's assessment about the subleading potential, they carried out hybrid computations of axial [53, 54] and vector [55, 56] currents, applying the latter to radiative neutron-proton capture. Tae-Sun does not get the recognition he deserves for this pioneering work, perhaps because he was partly out of sight. Mannque did what he could to promote both Tae-Sun's and my work, inviting me not only to speak at the $1993 \mathrm{ECT}^{*}$ workshop on chiral symmetry but also to lecture at a summer school in Seoul in June 1994. The warm Korean hospitality helped me keep going as a young postdoc.

In order to convince skeptics, we were always on the lookout for opportunities to go beyond conventional models and, as I was finishing up my $\mathrm{PhD}$, Lanny drew my attention to problems in the theoretical explanation for new data on neutral-pion production in nucleon-nucleon collisions near threshold. This was closely related to my qualifier problem, and Seattle was the right place to land on: Jerry had done the benchmark calculation for this process [57]. With Jim and Tom Cohen, who happened to be visiting on sabbatical, we immediately realized [58] that the power counting had to be modified on account of the larger typical momentum $\mathcal{O}\left(\sqrt{m_{\pi} m_{N}}\right)$ required to create a pion from a two-nucleon state. As a consequence of a vanishing LO and other cancellations, the bulk of the cross-section arises from contributions that are relatively unknown, which we modeled by heavier-meson exchange [59]. With a visitor from Brazil, Carlos da Rocha, Jerry and I marched on to charged-pion production, which is less pathological but still hard to describe [60]. As then-postdoc Chris Hanhart showed, $P$ waves are better behaved and can be used to constrain the three-nucleon force [61. In parallel, Kuniharu Kubodera and Fred Myhrer, with whom I had discussed EFT after they drove to Duke University during my 1994 visit, analyzed neutral-pion production with the standard power counting designed for momenta $\mathcal{O}\left(m_{\pi}\right)$ 62]. We eventually collaborated in order to understand the peculiarities of this reaction 63. The biggest success was tracking the effects of chargesymmetry violation from the quark mass difference. We enlisted Jouni Niskanen for a prediction of the forward-backward asymmetry in $p n \rightarrow d \pi^{0}$ [64], which was confirmed experimentally within a couple of years at TRIUMF 65. We then extended this work to the more complicated $d d \rightarrow \alpha \pi^{0}$, which was detected for the first time at the level we expected [66]. We later improved the treatment of the various contributions to this complicated process [67, 68].

Within a few years of the first publications, the case had been made, it seemed to me [69], for nuclear EFT as a foundation for the whole low-energy nuclear physics: many qualitative aspects of traditional nuclear physics had been explained, a decent (but not spectacular) fit to two-nucleon data obtained, useful currents derived, and predictions 
confirmed experimentally. Yet, the fun was just beginning.

\section{The Age of the Cockroaches}

Until about 1996, nuclear EFT felt like a lonely place. I believe I have already mentioned everybody worldwide who had an active interest in the subject, and most of them were focused on specific processes rather than a comprehensive approach. Then, catalyzed by the challenges raised in Refs. [70, 71, 72, 73] and confronted in two workshops (1998 in Pasadena [74] and 1999 in Seattle [75]), a small nuclear EFT community started to form. There are plenty of anecdotes from this era, but I save them for when we celebrate it.

1999 witnessed the clash in an epic Trento workshop (co-organized with Rob, Paulo Bedaque, and Ben Gibson) between young effective-field theorists and established potential modelers, who traded the war names of "cockroaches" and "dinosaurs". Trento was the place where many in the nuclear theory community had the first opportunity to see EFT in depth. EFT was increasingly becoming part of the mainstream. The first signal was Jim's work with the Nijmegen group [76, 77], who successfully replaced the one-meson-exchange interaction in their state-of-the-art phase-shift analysis [32] by the long-range part of chiral TPE. The process picked up pace with the improvements made in the Deltaless potential by the Bochum-Jülich group and their collaborators [78, 79], and then accelerated dramatically after the landmark fit to two-nucleon data by David Entem and Rupert Machleidt [80]. These developments came at a time when ab initio many-body methods were mature enough to welcome, and soon test, two- and three-nucleon forces having connections with QCD. Nowadays, rare is the ab initio calculation that does not use a chiral potential as input. We have reached the point where predictions for a wide variety of nuclei can be confronted with data and used to infer the pros and cons of the input potentials [14].

As great as this advance is, it has been patterned on the early work, with nuclear potentials and currents derived from EFT to some order but then treated as black boxes, as if they had originated in just another model. Since 1996, however, we have been discovering the various ways in which this approach is at odds with the tenets of EFT:

- In EFT, there is a hierarchy among the components of the potential. LO should capture the essential physics, for example generating bound states and saturation at momenta (or densities) within the regime of the theory. Subleading orders, on the other hand, should be amenable to perturbation theory. Because EFT potentials are increasingly singular with order, it is not guaranteed that it makes sense to solve the Schrödinger equation exactly with a potential truncated at some subleading order. I discuss the insistence on iterating what does not need to, and probably cannot, be iterated - which I like to call the nuclear Cohen syndrome in homage to Tom, who identified the disease - in Sec. 4.

- The attitude ingrained in the nuclear community is that one needs to nail the two-nucleon potential down perfectly up to some high energy before considering 
three-body forces, the form of which is difficult to guess phenomenologically. Aptly called $\chi^{2}$ paranoia by Rupert, this attitude persists today in the effort to go to very high order in the chiral two-nucleon potential — see Rupert's perspective in this celebratory volume [81]. There is of course nothing wrong with trying to describe the two-nucleon system better, but one should keep in mind that the EFT expansion is in a ratio of momentum scales, not in the number of nucleons per se. It might be possible to get a good description of nuclei including the few-body forces that are prescribed by the theory without attaining at the same order a perfect fit to two-nucleon scattering all the way to the pion-production threshold. This is the topic of Sec. 5 .

- EFT is a theory of everything (in low-energy nuclear physics): the same remarks about the potential apply to the kernels of reactions involving external probes like pions and photons, see Sec. 6.

- The fact that pions exist does not mean that they need to appear explicitly in the theory. But if they do, then another low-energy degree of freedom — the Delta isobar — should also be important. Delta denialism is confronted in Sec. 7.

\section{Renormalization and Naturalness}

Steve's papers do not betray much worry about renormalization, but that was certainly in the air in Austin. At some point while walking through campus I had a panic attack when it downed on me that the OPE tensor force might lead to extreme regulator dependence in the solution of the Schrödinger equation. I calmed down when I convinced myself that just looking at the diagrams in perturbation theory was not a good guide for what happened with the full, nonperturbative solution. In his second paper [4], Steve solved analytically the case of a pure, non-derivative contact interaction, from which it was clear that renormalization was different from that of the perturbative series. The problem was renormalizable despite the singularity of the Dirac delta function, giving us hope that it would be so also with the additional OPE at LO. If that was the case, then we expected a truncation of the potential at some order to yield all terms of the truncation of the amplitude at the same order, plus a subset of small, harmless higher-order contributions.

In addition to the finite number of irreducible loops in a truncation of the potential, the amplitude contains also the reducible loops subsumed in the solution of the LippmannSchwinger equation — or, equivalently, the Schrödinger equation. A loop requires a regulator to suppresses high momenta. The favorite technique is dimensional regularization, but it can only be implemented when the calculation is reduced analytically to a finite number of loops - for example, the contact interaction Steve solved. In an exact numerical solution of the Schrödinger equation, as needed for LO OPE, one is effectively summing up an infinite number of loops. A loop is a loop, so it is natural to use for the potential and the dynamical equation the same cutoff regulator: loop integrals are defined with a function $f(p / \Lambda)$ of momentum $p$ parametrized by a single number $\Lambda$, such 
that $f(0)=1$ but $f(\infty)=0$. Back then [30, 31, we chose a Gaussian function of relative momentum to make the potential as local as we could while killing all potential divergences. This regulator also allowed us to check parts of the long-range potential against Rijken's papers [82, 83]. Soon, the potential was recalculated in dimensional regularization [84, 85], which is sufficient for perturbation theory in high partial waves where the centrifugal barrier prevents two low-energy nucleons from getting close. The long-range part of the potential [76, 77] is the same, but its overall form much simpler. Most subsequent work adopted different regularizations for irreducible and reducible loops, with cleaner expressions but dirtier regulator dependence.

The model independence of EFT - hence its claim to reproduce QCD at low energies - is only guaranteed for observables, and only if the effects of the arbitrary regulator are removed by renormalization. How can they be removed? In pre-history, it was thought necessary to send the cutoff $\Lambda$ to infinity, but in EFT it is sufficient that its effects be suppressed by positive powers of $Q / \Lambda$ for $Q \sim M_{\mathrm{lo}} \ll M_{\mathrm{hi}}$, where $M_{\mathrm{lo}}$ stands for the low-energy scales and $M_{\text {hi }}$ for the breakdown scale. The aim of an EFT is to construct the most general $S$ matrix, with a power counting that allows for controlled truncations at each order $n$ :

$$
S^{(n)}\left(Q \sim M_{\mathrm{lo}}\right)=1+\sum_{\nu=0}^{n}\left(\frac{Q}{M_{\mathrm{hi}}}\right)^{\nu} F^{(\nu)}\left(\frac{Q}{M_{\mathrm{lo}}} ; \gamma^{(\leq \nu)}\right)+\mathcal{O}\left(\frac{Q^{n+1}}{M_{\mathrm{hi}}^{n+1}}, \frac{Q^{n+1}}{M_{\mathrm{hi}}^{n} \Lambda}\right)
$$

where the $F^{(\nu)}$ s are functions generated at order $\nu$ by the dynamics of the explicit degrees of freedom and $\gamma^{(\leq \nu)}$ stands for the LECs appearing up to that order. Then, for sufficiently large $\Lambda$ - not necessarily only for $\Lambda \rightarrow \infty$ [86] - regulator effects can be made no larger than the next order in the EFT expansion, which is of relative size $\mathcal{O}\left(Q / M_{\text {hi }}\right)$. When $\mathrm{LO}$ is nonperturbative often we have to check renormalizability numerically, which might require values well above $M_{\text {hi }}$ to rule out mild dependence, such as logarithmic.

In order to achieve $\Lambda$-independent $F^{(\nu)} \mathrm{s}$ in Eq. (11), enough LECs need to be present at each order to remove the non-negative powers of $\Lambda$ generated by the loops. Naturalness [87, 88] is the idea that a LEC needed to absorb the cutoff dependence reflects sensitivity to short-range physics at the same order. The LEC then should not only remove the offending powers of $\Lambda$ from observables but also have an unknown finite part. The latter is to be determined by matching to either the underlying theory or to data. The NDA [16, 17] on which Weinberg's power counting is based was derived by looking at scattering amplitudes perturbatively, renormalizing them with the necessary LECs, and assuming naturalness. Steve applied this idea to the potential, which he argued has a perturbative expansion. The hope in early days was that the nonperturbative part of the calculation would not generate further non-negative powers of $\Lambda$ and thus not affect the NDA-based ordering of interactions.

With our local regulator [30, 31, every contact interaction contributed to every wave. To facilitate the comparison with one-boson exchange models, we did not use in the fit the minimum number of nine two-nucleon LECs that appeared up to $\mathrm{N}^{3} \mathrm{LO}$ according to NDA [5], but a redundant set of 18. Starting with Ref. [78], the minimal set of LECs and nonlocal regulators for the dynamical equation have been frequently employed, 
allowing for much easier fits partial wave by partial wave. But, all in all, in 1996 we had 26 parameters and the fitting procedure needed at each cutoff was laborious. The qualitatively similar results for the Deltaful $\mathrm{N}^{3} \mathrm{LO}$ potential at the three cutoffs of $\simeq 500$, 780 and $1000 \mathrm{MeV}$ we could reasonably handle [31] gave us some reassurance that the problem was renormalizable. No panic attack.

It did not take long, though, for David, with Martin Savage and Mark Wise, to point out that there is a problem: in the two-nucleon ${ }^{1} S_{0}$ channel, one can see semi-analytically [70, 89, 90, that at LO in the original power counting an $m_{\pi}^{2} \ln \Lambda$ dependence is generated by the dynamical equation which cannot be absorbed in the chiral-symmetry-conserving LEC present at that order. The four-nucleon-field interaction that removes this cutoff dependence breaks chiral symmetry explicitly in the same way as the average quark mass in QCD, that is, as a component of a chiral four-vector. As a consequence, it is accompanied, unlike the chiral-symmetric interaction present at LO, by an infinite tower of additional interactions involving an even number of pions. It should only appear at $\mathrm{N}^{2} \mathrm{LO}$ according to NDA, so clearly NDA - the cornerstone of the approach — is being violated.

For a while the wishful thinking was that this could be an isolated failure, or at most limited to chiral-symmetry breaking. If so, then some contributions would be enhanced, but generally only in higher orders - for example, in two-nucleon scattering when the additional pions formed loops - and/or in less fundamental processes - like pion scattering on the three-nucleon system. But then came the work of Daniel, Tom, and Silas [71, 72, 73], which showed that, when pion interactions are turned off, Weinberg's prescription does not work at subleading orders. The importance of crises in scientific progress is well documented, and a crisis this was.

The community has been split ever since. Most people decided to simply choose regulators (spectral-function regularization, "super Gaussian" functions, different cutoff values for each partial wave, etc.) that allow for the best data fitting, along the way giving up on the idea of a priori error estimates. This attitude has produced numerous phenomenological successes [12, 13] as well as some failures which are gaining increased attention. Effective-field theorists have, in contrast, fussed over the issue of renormalization.

It became clear soon that the nuclear Cohen syndrome can be traced to treating the subleading parts of the potential nonperturbatively [91, 92, 93, 94, 95, 96]. LO does require an exact solution to produce the $S$-matrix poles we associate with bound states and resonances, but subleading interactions should in principle be incorporated in distortedwave perturbation theory. In the case of purely contact interactions, naturalness at LO implies that the poles are outside the range of the theory. Once the fine tuning required for the shallow deuteron and ${ }^{1} S_{0}$ virtual state is incorporated, naturalness still allows for the construction of a consistent power counting for subleading interactions. This Pionless EFT [15] works well at momenta below the pion mass.

The first important lesson from Pionless EFT is thus that, while one can expand the potential, each term enters the expansion of the amplitude differently. A FAQ is why, if a correction is, well, a correction, can it not be treated nonperturbatively? The answer is that in general only for regular interactions can one do this while preserving model independence. For the increasingly singular EFT interactions, what is small is the sum of 
contributions at a given subleading order in the amplitude. There can be cancellations, the magnitude of individual contributions being regulator dependent. When one solves exactly for a subleading truncation of the potential, one includes in the amplitude many loops without the LECs that would cancel their cutoff dependence. Sometimes - for example, implicitly when using as input to LO Chiral EFT data at physical pion mass rather of their chiral-limit values - this can be done without affecting renormalization. However, resumming ("unitarizing") a truncated EFT potential will usually generate regulator dependence: the partial subset of higher-order corrections is not harmless. Unless resummation with a specific regulator can be somehow justified, the resulting regulator dependence must be considered a model for the short-range physics. The question that should be frequently asked instead is, if corrections are indeed corrections, should they not be amenable to distorted-wave perturbation theory, regardless of regularization issues? There are no tests that this is the case for NDA-based chiral potentials.

The second important lesson for Chiral EFT is the power counting of factors of $4 \pi$ in reducible loops. In addition to the $m_{N} / Q$ enhancement noticed by Weinberg, there is a second enhancement of $4 \pi$. For example, one should count

$$
\text { reducible two-nucleon loop } \sim \frac{m_{N} Q}{4 \pi},
$$

instead of the $Q^{2} /(4 \pi)^{2}$ for an irreducible loop. In Pionless EFT the LO contact interaction is fine tuned to be (after renormalization, of course)

$$
\text { nonderivative contact interaction } \sim \frac{4 \pi}{m_{N} M_{\mathrm{lo}}},
$$

so the LO loops in the Lippmann-Schwinger equation are an expansion in $Q / M_{\mathrm{lo}}$. The dynamical equation needs to be solved nonperturbatively and can lead to poles at momenta $\kappa_{A}=\mathcal{O}\left(M_{\mathrm{lo}}\right)$. OPE, on the other hand, has a strength

$$
\text { one-pion exchange } \sim \frac{4 \pi}{m_{N} M_{N N}},
$$

where $M_{N N} \equiv 16 \pi f_{\pi}^{2} / g_{A}^{2} m_{N}$ [92, 97, 95], with $f_{\pi} \simeq 93 \mathrm{MeV}$ the pion decay constant and $g_{A} \simeq 1.27$ the LO pion-nucleon coupling. The OPE ladder is thus a series in $Q / M_{N N}$.

David, Martin, and Mark built on Pionless EFT by suggesting that $M_{N N}$, which numerically is $\approx 300 \mathrm{MeV}$, be treated as a high-energy scale [92, 97, 95]. In this case LO is formally the same as in Pionless EFT, pions can be treated perturbatively, and no renormalization issues arise from pion exchange. Unfortunately, convergence of perturbative pions deteriorates rapidly for momenta above $100 \mathrm{MeV}$ or so [98, 99], and at physical pion mass this version of Chiral EFT does not greatly improve over Pionless EFT.

In hindsight, such a failure is not unexpected: from naturalness, $m_{N}=\mathcal{O}\left(M_{\mathrm{QCD}}\right)$, $f_{\pi}=\mathcal{O}\left(M_{\mathrm{QCD}} / 4 \pi\right)$, and $g_{A}=\mathcal{O}(1)$, so that $M_{N N}=\mathcal{O}\left(f_{\pi}\right)$. The two reducible-loop enhancements lead to the expectation that OPE needs to be resummed and generates poles with momenta

$$
\kappa_{A}=\mathcal{O}\left(f_{\pi}\right)=\mathcal{O}\left(\frac{M_{\mathrm{QCD}}}{4 \pi}\right),
$$


in the absence of fine tuning. While this is somewhat larger than the binding momentum of the deuteron $\left(\kappa_{2} \simeq 45 \mathrm{MeV}\right)$, it is not too far off for heavier nuclei. Of course this argument is very simplistic as it does not take into account the range of the potential given by the pion mass. But, as Steve pointed out [4], without the additional enhancement by $4 \pi$ one would have to consider $m_{N}$ as particularly large, in fact $\mathcal{O}\left(M_{\mathrm{QCD}}^{2} / M_{\mathrm{lo}}\right)$ [31]. This dated argument underlies most subsequent work with chiral potentials [12, 13], where nucleon recoil and relativistic corrections are taken to be suppressed by powers of $M_{\mathrm{QCD}}^{2}$ and the origin of $M_{\mathrm{lo}}$ is left in the air. In contrast, Eq. (5) suggests $M_{\mathrm{lo}}=\mathcal{O}\left(f_{\pi}\right)$ and explains why $\kappa_{A} \ll M_{\mathrm{QCD}}$, a mystery when one does not take into account naturalness in the presence of spontaneous symmetry breaking.

But can we renormalize the tensor part of OPE, which does not contribute to ${ }^{1} S_{0}$ ? Memories of my Austin panic attack haunted me when Martin argued in talks - for example, Ref. 97 — that successive loops in the OPE ladder would require LECs from interactions with more and more derivatives. So it was high time to settle this issue in the nonperturbative context. It is remarkable that the LO in Weinberg's power counting is renormalizable in the coupled ${ }^{3} S_{1^{-}}{ }^{3} D_{1}$ channels after all [100, 89, 101, 102]. Unfortunately, this is not true in the higher waves where the OPE tensor force is attractive [103, 104] but Weinberg's power counting prescribes no LO LECs. The solution is to treat pions at LO only in the lower waves - besides $S$ waves, ${ }^{3} P_{0}$ and perhaps a couple more where it is nonperturbative, together with the LECs that guarantee renormalization [103]. OPE in higher waves is suppressed by powers of the orbital angular momentum due to the centrifugal barrier [105, 106, 99] and can be included in higher orders. More singular corrections can indeed be renormalized in distorted-wave perturbation theory [107, 108, 109.

Reasonable agreement with data is found [110, 111, 112, 113, 90, 114], but clearly improvements are needed, especially in the ${ }^{1} S_{0}$ channel [89, 115, 116]. Fixing the chiral expansion opens up the possibility of matching to lattice QCD data at pion masses where Chiral EFT converges. A possible scenario [89] is that the deuteron and ${ }^{1} S_{0}$ virtual state accidentally cross zero energy close to physical pion mass, a description of fine tuning in terms of quark masses analogous to that of atomic Feshbach resonances in terms of an external magnetic field.

But these ideas also have consequences beyond the two-nucleon system...

\section{$5 \quad$ Few-Body Forces}

In the first paper [3] Steve did not realize that there is an additional suppression for few-nucleon forces with respect to two-nucleon forces. He was quick to rectify this in his second paper [4], where he argued for a $Q^{2} / M_{\mathrm{QCD}}^{2}$ suppression for each additional nucleon involved in the potential. This argument was based on a particular counting of factors of $4 \pi$. Already in 1996, Jim proposed [117] a different counting where the penalty is instead $Q / M_{\mathrm{QCD}}$. Jim based his argument on his pre-EFT ideas [33, 34], but it is also what results when one counts $4 \pi \mathrm{s}$ in reducible loops as in Pionless EFT [15]. In either case, the 
relative suppression of few-nucleon forces was an early qualitative success of Chiral EFT, as it matched experience with phenomenological models that include pion exchange.

However, the relative importance of few-nucleon forces is not well established in Chiral EFT. It is worrisome that saturation in nuclear matter with NDA-based chiral potentials seems to occur in the low-energy region only at orders where three-body forces are included [118, 119]. A careful uncertainty quantification [120] indicates that NDA-based chiral potentials have such large errors at LO and NLO that any equilibrium density is possible. There is no guarantee that the equilibrium point of symmetric nuclear matter is within Chiral EFT but, if it is, it must appear in its region of applicability already at LO, to be moved only slightly by higher orders. (Nobody would — or at least should — buy into an expansion where the alpha particle, say, does not have at LO a definite binding momentum below the breakdown scale.)

In fact, in Pionless EFT [15] a three-body force is required at LO to achieve renormalization in the three-body system [121, 122, 123], which then explains Efimov physics [124]. It is sufficient for renormalization in larger systems also, without the need for four-body forces [125, 126] until one reaches NLO [127]. A good description of systems with $A=3,4$ nucleons emerges starting with two-body unitarity at LO [128, 129, 130].

This example of proper nuclear structure without a fit to high-energy two-nucleon data has not been sufficient to cure $\chi^{2}$ paranoia. But it has led Alejandro Kievsky and collaborators to propose that three-nucleon forces should be LO also in Chiral EFT [131, 132]. They have pointed out that the description of three-nucleon data with NDAbased chiral potentials is relatively poor even at the high orders where the two-nucleon system is essentially perfectly fitted. Adding a three-body force improves on Weinberg's LO significantly for $A=3,4$ [131] and yields nuclear saturation [132. The difference with Pionless EFT is that there is no evidence the three-nucleon force is needed for LO renormalization once the two-nucleon system is renormalized [103, 133, 134]. In this case, results are reasonable for $A=3,4$ up to NLO without three-body forces [133, 134], but at LO $p$-shell nuclei are unstable [134] and nuclear matter underbound [135] for the limited cutoff values that are accessible. Jerry Yang and collaborators have suggested recently [136] that three- and four-nucleon forces might be enhanced by combinatorial factors of $A$. I do not think we have heard the last of enhanced few-nucleon forces.

\section{External Probes}

Concern existed for a long time about the consistency between reaction kernels and potentials. It was hoped that Weinberg's hybrid approach would capture the most important physics, but we were aware that it would have to be replaced as higher precision was sought. Already in our first reaction paper [45], we used both chiral [30] and Bonn [137] wavefunctions in an attempt to gauge the uncertainties caused by the mismatch between potential and kernel.

One issue is that observables must be independent of the choice of fields, which means they should be obtained consistently from the same Lagrangian. Model independence also 
requires proper renormalization, just as for scattering amplitudes involving no external probes. One can expand the long-range part of a kernel with Weinberg-type counting, but the sizes of short-range interactions are not necessarily given by NDA. Power counting is for the amplitude, and the LECs of short-range interactions involving external probes might also be enhanced by renormalization running. That this is indeed the case was shown by Daniel and Manolo Pavón Valderrama [138].

A concrete example is neutrinoless double-beta decay, a lepton-number-violating process where two neutrons change into two protons and two electrons. It is expected to be driven by the exchange of a Majorana neutrino, whose tiny mass is generated by the lowest-dimensional (dimension-five) operator in the Standard Model EFT [139]. The most important contribution comes from the ${ }^{1} S_{0}$ channel, where its renormalization is similar to OPE's [70, 89, 90]. The consequence is that a short-range lepton-number-violating interaction is needed at LO [140, 141, but no new interaction appears at NLO [141]. The corresponding LEC can be estimated from the isospin-violating electromagnetic interactions to which it is related by chiral symmetry [140, 141], a procedure that is consistent from the perspective of an expansion in the inverse number of colors [142]. With such an estimate, the short-range contribution in light nuclei is indeed comparable to that of the long-range mechanism [140, 141]. Its first inclusion in an ab initio calculation for an experimentally relevant nucleus has confirmed its potential importance [143] and a strategy exists to determine its LEC directly from lattice QCD [144].

Unfortunately, the broader consequences of the Pavón-Phillips work [138] do not appear to be fully appreciated in the community. Moreover, the implications of an improved counting of $4 \pi \mathrm{s}$ for the main observation in Steve's third paper [6] - the suppression of few-body reaction kernels, which are the EFT version of "meson-exchange currents" have not been explored.

\section{Delta Isobar}

I learned about the importance of the Delta isobar while preparing for my qualifying exam and, later, reading Gerry [11] and Karl, Rupert, and Charlotte Elster [137]. The Delta's prominence at relatively small energies comes from the Delta-nucleon mass difference $\Delta \equiv m_{\Delta}-m_{N} \ll M_{\mathrm{QCD}}$. If we do not want to limit the convergence of the EFT too severely, $\Delta$ should be treated as a low-energy scale and the Delta kept as an explicit degree of freedom.

Steve was pleased to hear that Carlos and I had already started including the Delta isobar when he reported that the local group made this suggestion during his visit to the University of Maryland in the early '90s. There existed an immense literature on the difficulties and ambiguities of a spin-3/2 field, but none are relevant in the nonrelativistic expansion. In the original submission of our first paper [5] we presented the two-nucleon potential up to $\mathrm{N}^{3} \mathrm{LO}$ with the Delta. It was an expansion in $Q / M_{\mathrm{QCD}}$ with $Q \sim m_{\pi} \sim \Delta$. Our referee was supportive except for the explicit Delta. One heard such an objection frequently in the early days, as many chiral perturbation theorists seemed to think of ChPT 
less as a specific EFT than as a special representation of QCD at vanishing momentum and pion mass - unable to handle an expansion parameter like $\Delta / M_{\mathrm{QCD}}$ which does not vanish in these limits, even if it is small in the real world. I was in a hurry to have the paper published before going into the postdoc job market, so rather than arguing we replaced the Delta components of the two-nucleon force by the leading Deltaless threenucleon force. We did consider the Delta explicitly in the potential we fitted to data later [30, 31], where it entered starting with the box, crossed box, and triangle TPE diagrams of rough size

$$
\text { Deltaful two-pion exchange } \sim \frac{4 \pi}{m_{N} M_{N N}} \frac{Q^{3}}{\Delta M_{\mathrm{QCD}}^{2}} .
$$

This is $\mathrm{N}^{2} \mathrm{LO}$ just as

$$
\text { Deltaless two-pion exchange } \sim \frac{4 \pi}{m_{N} M_{N N}} \frac{Q^{2}}{M_{\mathrm{QCD}}^{2}}
$$

if $\Delta$ is considered a low-energy scale, but it enters only at $\mathrm{N}^{3} \mathrm{LO}$ for $\Delta$ taken as a highenergy scale. Integrating out the Delta, as done by subsequent generations of chiralpotential builders, jeopardizes the convergence pattern of Chiral EFT.

At about the same time, Elizabeth Jenkins and Aneesh Manohar demonstrated the importance of the Delta in ChPT [145], with the systematic expansion in $\Delta / M_{\mathrm{QCD}}$ exploited in subsequent papers such as Ref. [146]. Later, when studied in further detail [147], this expansion was rebranded the "small-scale expansion" as a nod to chiral perturbation theorists. Perhaps thanks to this move, the Delta was eventually accepted in ChPT, a milestone being the work by Ulf and Nadia Fettes on pion-nucleon scattering below the Delta peak [148]. Around the Delta peak, power counting must be modified to account for the cancellation between energy and $\Delta$ in $s$-channel Delta propagation. This was done first for Compton scattering [149] taking $m_{\pi} \sim \Delta^{2} / M_{\mathrm{QCD}}$ and later for pion-nucleon scattering [150] with the more standard $m_{\pi} \sim \Delta$. Beyond the Delta peak, the Roper resonance becomes important [151, 152].

These developments of course only reinforced the motivation to include the Delta explicitly in the nuclear potential and reaction kernels. Over the years I had many screaming matches with Vijay Pandharipande, particularly when he visited Caltech in the late '90s and insisted on the importance of the small hole in a deuteron wavefunction "donut". But one thing we could agree on was the Delta. Vijay was convinced the Delta was crucial, particularly through the contribution (NLO in Friar's counting) of the Fujita-Miyazawa three-nucleon force [153]. One cannot but admire Vijay's legendary drive when he transmitted notes - a chapter from a book he was writing - to Daniel while he was away from home pursuing treatment at a hospital. Daniel and I translated Vijay's point into EFT language while we were at an INT workshop in October 2004. In what I have been told was Vijay's last paper [154], we showed in pretty concrete terms, I thought, how omitting the low-lying Delta leads to relatively large errors in Chiral EFT: to relate pion-nucleon scattering to the TPE three-nucleon force, one extrapolates in energy by at least $m_{\pi}$, thus generating errors $\mathcal{O}\left(m_{\pi}^{2} / \Delta^{2}\right)$ in the three-nucleon force when the Delta is not included 
explicitly. Of course these errors can be partly corrected two orders down the expansion but including all contributions at such a high order is, well, a tall order.

The successes of Pionless EFT [15] question whether pion propagation is as important for nuclear physics as previously believed. But of course including the pion explicitly should increase the EFT range, and in Chiral EFT there is no fundamental difficulty in accounting for the Delta as well. Still, despite the evidence for the Delta's importance from pre-EFT nuclear physics, from the first chiral potentials, and from ChPT, only recently has the Delta been taken seriously again in chiral potentials. As expected, it improves the description from light nuclei [155, 156] to nuclear matter [157, 158]. Unfortunately, Delta effects are not well studied in renormalizable Chiral EFT.

\section{Conclusion}

Hopefully these reminiscences give a glimpse of the many contributions to the development of news ideas which are often not apparent in the paper trail. The conceptual evolution of nuclear EFTs is particularly hard to follow since only a very selected type of paper has percolated through the nuclear community - one where the notions of renormalization, naturalness, and power counting for observables play second fiddle to potentials and kernels derived from an unquestioned recipe and then fitted to data at high orders. Only now are $\chi^{2}$ paranoia, Cohen syndrome, and Delta denialism losing their grip, see e.g. Ref. [159.

In retrospect, perhaps it was this that Gerry was warning us about back in the early '90s. The root of the failure of Steve's original recipe is the nonperturbative renormalization of singular interactions [160, 161], in particular the tensor force [103] that comes from the pions' derivative couplings and, thus, from spontaneous chiral-symmetry breaking. In phenomenological models (for example, Ref. [137]) the rho meson is assumed to have a tensor coupling which is adjusted to largely cancel OPE's tensor force at short distances. The need for such a cancellation had in fact been considered indirect evidence for the existence of the rho meson [162]. In Chiral EFT the cancellation is accomplished by LECs, not all of which obey NDA - probably what Mannque means by "not naturally suppressed" in his contribution to this volume [163]. It is not surprising in hindsight that the leading-order potential without the appropriate interactions is considered so bad by practitioners that most papers only show results from higher orders. In contrast, the ordering of the short-range interactions that control the tensor force does follow from naturalness in the nonperturbative context - just not from a perturbative expansion that does not in any case apply to the momenta of interest.

The research set in motion by the original papers [3, 9, 4, 5, 6] has achieved the longstanding goal of renormalization in nuclear structure and reactions, highlighting along the way the roles played by few-body forces and the Delta isobar — not to mention sparking the creation of Pionless and Halo/Cluster EFTs. This is nothing to sneeze at: it heals the scission between nuclear and particle physics inflicted by the failure of renormalization in the pion theories of the '50s. But the task of constructing a consistent Chiral EFT is 
far from complete, as renormalization is a necessary but insufficient constraint on power counting. For more on where we are in this process - but without the anecdotes - see Refs. [164, 165].

\section{Acknowledgments}

I thank Alejandro Kievsky for prodding me to collect my reminiscences here, and Silas Beane and Daniel Phillips for helping me recollect them. I am also thankful to Alejandro and Daniel for comments on the manuscript. This material is based upon work supported in part by the U.S. Department of Energy, Office of Science, Office of Nuclear Physics, under award DE-FG02-04ER41338.

\section{References}

[1] S. Weinberg, The First Three Minutes: A modern view of the origin of the universe, Basic Books (1977).

[2] S. Weinberg, Physica A 96 (1979) 327.

[3] S. Weinberg, Phys. Lett. B 251 (1990) 288.

[4] S. Weinberg, Nucl. Phys. B 363 (1991) 3.

[5] C. Ordóñez and U. van Kolck, Phys. Lett. B 291 (1992) 459.

[6] S. Weinberg, Phys. Lett. B 295 (1992) 114.

[7] S.R. Beane and U. van Kolck, Phys. Lett. B 328 (1994) 137.

[8] G.E. Brown and M. Rho, Phys. Rev. Lett. 66 (1991) 2720.

[9] M. Rho, Phys. Rev. Lett. 66 (1991) 1275.

[10] G.E. Brown, Comments Nucl. Part. Phys. 4 (1970) 140.

[11] G.E. Brown, Comments Nucl. Part. Phys. 5 (1972) 6.

[12] E. Epelbaum, H.-W. Hammer, and U.-G. Meißner, Rev. Mod. Phys. 81 (2009) 1773.

[13] R. Machleidt and D.R. Entem, Phys. Rept. 503 (2011) 1.

[14] H. Hergert, Front. in Phys. 8 (2020) 379.

[15] H.-W. Hammer, S. König, and U. van Kolck, Rev. Mod. Phys. 92 (2020) 025004.

[16] A. Manohar and H. Georgi, Nucl. Phys. B 234 (1984) 189. 
[17] H. Georgi, Phys. Lett. B 298 (1993) 187.

[18] V. Bernard, N. Kaiser, and U.-G. Meißner, Int. J. Mod. Phys. E 4 (1995) 193.

[19] S. Weinberg, Eur. Phys. J. H 46 (2021) 6.

[20] S. Weinberg, The Quantum Theory of Fields. Vol. 1: Foundations, Cambridge University Press (2005).

[21] S. Weinberg, The Quantum Theory of Fields. Vol. 2: Modern applications, Cambridge University Press (2013).

[22] S. Weinberg, The Quantum Theory of Fields. Vol. 3: Supersymmetry, Cambridge University Press (2013).

[23] D.B. Kaplan, Nucl. Phys. B 260 (1985) 215.

[24] H. Georgi, D.B. Kaplan, and L. Randall, Phys. Lett. B 169 (1986) 73.

[25] H. Georgi and L. Randall, Nucl. Phys. B 276 (1986) 241.

[26] E.E. Jenkins and A.V. Manohar, Phys. Lett. B 255 (1991) 558.

[27] L. Ray, Phys. Rev. C 35 (1987) 1072.

[28] U.L. van Kolck, "Soft Physics: Applications of Effective Chiral Lagrangians to Nuclear Physics and Quark Models", PhD dissertation, University of Texas at Austin (1993), UMI-94-01021.

[29] R.A. Arndt, J.S. Hyslop III, and L.D. Roper, Phys. Rev. D 35 (1987) 128.

[30] C. Ordóñez, L. Ray, and U. van Kolck, Phys. Rev. Lett. 72 (1994) 1982.

[31] C. Ordóñez, L. Ray, and U. van Kolck, Phys. Rev. C 53 (1996) 2086.

[32] V.G.J. Stoks, R.A.M. Klomp, M.C.M. Rentmeester, and J.J. de Swart, Phys. Rev. C 48 (1993) 792.

[33] J.L. Friar, Annals Phys. 104 (1977) 380.

[34] S.A. Coon and J.L. Friar, Phys. Rev. C 34 (1986) 1060.

[35] U. van Kolck, Phys. Rev. C 49 (1994) 2932.

[36] J.L. Friar, D. Hüber, and U. van Kolck, Phys. Rev. C 59 (1999) 53.

[37] D. Hüber, J.L. Friar, A. Nogga, H. Witała, and U. van Kolck, Few-Body Syst. 30 (2001) 95 .

[38] U. van Kolck, Few-Body Syst. Suppl. 9 (1995) 444. 
[39] U. van Kolck, J.L. Friar, and J.T. Goldman, Phys. Lett. B 371 (1996) 169.

[40] U. van Kolck, M.C.M. Rentmeester, J.L. Friar, J.T. Goldman, and J.J. de Swart, Phys. Rev. Lett. 80 (1998) 4386.

[41] J.L. Friar and U. van Kolck, Phys. Rev. C 60 (1999) 034006.

[42] J.L. Friar, U. van Kolck, G.L. Payne, and S.A. Coon, Phys. Rev. C 68 (2003) 024003.

[43] J.L. Friar, U. van Kolck, M.C.M. Rentmeester, and R.G.E. Timmermans, Phys. Rev. C 70 (2004) 044001.

[44] J.L. Friar, G.L. Payne, and U. van Kolck, Phys. Rev. C 71 (2005) 024003.

[45] S.R. Beane, C.-Y. Lee, and U. van Kolck, Phys. Rev. C 52 (1995) 2914.

[46] S.R. Beane, V. Bernard, T.-S.H. Lee, U.-G. Meißner, and U. van Kolck, Nucl. Phys. A 618 (1997) 381.

[47] J.C. Bergstrom et al., Phys. Rev. C 57 (1998) 3203.

[48] S.R. Beane, M. Malheiro, D.R. Phillips, and U. van Kolck, Nucl. Phys. A 656 (1999) 367.

[49] S.R. Beane, M. Malheiro, J.A. McGovern, D.R. Phillips, and U. van Kolck, Phys. Lett. B 567 (2003) 200 [erratum: Phys. Lett. B 607 (2005) 320].

[50] S.R. Beane, M. Malheiro, J.A. McGovern, D.R. Phillips, and U. van Kolck, Nucl. Phys. A 747 (2005) 311.

[51] H.W. Grießhammer, J.A. McGovern, D.R. Phillips, and G. Feldman, Prog. Part. Nucl. Phys. 67 (2012) 841.

[52] D.R. Phillips, "What Hath Weinberg Wrought? Reflections on what Weinberg's papers on 'Nuclear Forces from Chiral Lagrangians' did and did not accomplish", arXiv:2107.03558 [nucl-th].

[53] T.-S. Park, D.-P. Min, and M. Rho, Phys. Rept. 233 (1993) 341.

[54] T.-S. Park, I.S. Towner, and K. Kubodera, Nucl. Phys. A 579 (1994) 381.

[55] T.-S. Park, D.-P. Min, and M. Rho, Phys. Rev. Lett. 74 (1995) 4153.

[56] T.-S. Park, D.-P. Min, and M. Rho, Nucl. Phys. A 596 (1996) 515.

[57] G.A. Miller and P.U. Sauer, Phys. Rev. C 44 (1991) R1725.

[58] T.D. Cohen, J.L. Friar, G.A. Miller, and U. van Kolck, Phys. Rev. C 53 (1996) 2661.

[59] U. van Kolck, G.A. Miller, and D.O. Riska, Phys. Lett. B 388 (1996) 679. 
[60] C.A. da Rocha, G.A. Miller, and U. van Kolck, Phys. Rev. C 61 (2000) 034613.

[61] C. Hanhart, U. van Kolck, and G.A. Miller, Phys. Rev. Lett. 85 (2000) 2905.

[62] B.-Y. Park, F. Myhrer, J.R. Morones, T. Meissner, and K. Kubodera, Phys. Rev. C 53 (1996) 1519.

[63] C. Hanhart, G.A. Miller, F. Myhrer, T. Sato, and U. van Kolck, Phys. Rev. C 63 (2001) 044002 .

[64] U. van Kolck, J.A. Niskanen, and G.A. Miller, Phys. Lett. B 493 (2000) 65.

[65] A.K. Opper et al., Phys. Rev. Lett. 91 (2003) 212302.

[66] E.J. Stephenson et al., Phys. Rev. Lett. 91 (2003) 142302.

[67] A. Gårdestig, C.J. Horowitz, A. Nogga, A.C. Fonseca, C. Hanhart, G.A. Miller, J.A. Niskanen, and U. van Kolck, Phys. Rev. C 69 (2004) 044606.

[68] A. Nogga, A.C. Fonseca, A. Gårdestig, C. Hanhart, C.J. Horowitz, G.A. Miller, J.A. Niskanen, and U. van Kolck, Phys. Lett. B 639 (2006) 465.

[69] U. van Kolck, Prog. Part. Nucl. Phys. 43 (1999) 337.

[70] D.B. Kaplan, M.J. Savage, and M.B. Wise, Nucl. Phys. B 478 (1996) 629.

[71] D.R. Phillips and T.D. Cohen, Phys. Lett. B 390 (1997) 7.

[72] D.R. Phillips, S.R. Beane, and T.D. Cohen, Annals Phys. 263 (1998) 255.

[73] S.R. Beane, T.D. Cohen, and D.R. Phillips, Nucl. Phys. A 632 (1998) 445.

[74] R. Seki, U. van Kolck, and M. J. Savage, Nuclear Physics with Effective Field Theory, World Scientific (1998).

[75] P.F. Bedaque, M.J. Savage, R. Seki, and U. van Kolck, Nuclear Physics with Effective Field Theory II, World Scientific (2000).

[76] M.C.M. Rentmeester, R.G.E. Timmermans, J.L. Friar, and J.J. de Swart, Phys. Rev. Lett. 82 (1999) 4992.

[77] M.C.M. Rentmeester, R.G.E. Timmermans, and J.J. de Swart, Phys. Rev. C 67 (2003) 044001.

[78] E. Epelbaum, W. Glöckle, and U.-G. Meißner, Nucl. Phys. A 671 (2000) 295.

[79] E. Epelbaum, A. Nogga, W. Glöckle, H. Kamada, U.-G. Meißner, and H. Witała, Phys. Rev. C 66 (2002) 064001.

[80] D.R. Entem and R. Machleidt, Phys. Rev. C 68 (2003) 041001. 
[81] R. Machleidt, Few-Body Syst. 62 (2021) 21.

[82] T.A. Rijken, Annals Phys. 208 (1991) 253.

[83] T.A. Rijken and V.G.J. Stoks, Phys. Rev. C 46 (1992) 73.

[84] N. Kaiser, R. Brockmann, and W. Weise, Nucl. Phys. A 625 (1997) 758.

[85] N. Kaiser, S. Gerstendörfer, and W. Weise, Nucl. Phys. A 637 (1998) 395.

[86] G.P. Lepage, "What is Renormalization?", arXiv:hep-ph/0506330 [hep-ph].

[87] G. 't Hooft, NATO Sci. Ser. B 59 (1980) 135.

[88] M.J.G. Veltman, Acta Phys. Polon. B 12 (1981) 437.

[89] S.R. Beane, P.F. Bedaque, M.J. Savage, and U. van Kolck, Nucl. Phys. A 700 (2002) 377.

[90] B. Long and C.-J. Yang, Phys. Rev. C 86 (2012) 024001.

[91] U. van Kolck, Lect. Notes Phys. 513 (1998) 62.

[92] D.B. Kaplan, M.J. Savage, and M.B. Wise, Phys. Lett. B 424 (1998) 390.

[93] D.B. Kaplan, "Power Counting and the Renormalization Group for an Effective Description of NN Scattering", in Ref. [74, arXiv:nucl-th/9804061 [nucl-th].

[94] U. van Kolck, "The Aleph", in Ref. [74].

[95] D.B. Kaplan, M.J. Savage, and M.B. Wise, Nucl. Phys. B 534 (1998) 329.

[96] U. van Kolck, Nucl. Phys. A 645 (1999) 273.

[97] M.J. Savage, "Including Pions", in Ref. [74], arXiv:nucl-th/9804034 [nucl-th].

[98] S. Fleming, T. Mehen, and I.W. Stewart, Nucl. Phys. A 677 (2000) 313.

[99] D.B. Kaplan, Phys. Rev. C 102 (2020) 034004.

[100] T. Frederico, V.S. Timóteo, and L. Tomio, Nucl. Phys. A 653 (1999) 209.

[101] D. Eiras and J. Soto, Eur. Phys. J. A 17 (2003) 89.

[102] M. Pavón Valderrama and E. Ruiz Arriola, Phys. Rev. C 72 (2005) 054002.

[103] A. Nogga, R.G.E. Timmermans, and U. van Kolck, Phys. Rev. C 72 (2005) 054006.

[104] M. Pavón Valderrama and E. Ruiz Arriola, Phys. Rev. C 74 (2006) 064004 [erratum: Phys. Rev. C 75 (2007) 059905]. 
[105] M.C. Birse, Phys. Rev. C 74 (2006) 014003.

[106] M. Pavón Valderrama, M. Sánchez Sánchez, C.-J. Yang, B. Long, J. Carbonell, and U. van Kolck, Phys. Rev. C 95 (2017) 054001.

[107] M.C. Birse, Phys. Rev. C 76 (2007) 034002.

[108] B. Long and U. van Kolck, Annals Phys. 323 (2008) 1304.

[109] M.C. Birse, Eur. Phys. J. A 46 (2010) 231.

[110] M. Pavón Valderrama, Phys. Rev. C 83 (2011) 024003.

[111] M. Pavón Valderrama, Phys. Rev. C 84 (2011) 064002.

[112] B. Long and C.-J. Yang, Phys. Rev. C 84 (2011) 057001.

[113] B. Long and C.-J. Yang, Phys. Rev. C 85 (2012) 034002.

[114] S. Wu and B. Long, Phys. Rev. C 99 (2019) 024003.

[115] B. Long, Phys. Rev. C 88 (2013) 014002.

[116] M. Sánchez Sánchez, C.-J. Yang, B. Long, and U. van Kolck, Phys. Rev. C 97 (2018) 024001.

[117] J.L. Friar, Few-Body Syst. 22 (1997) 161.

[118] C. Drischler, K. Hebeler, and A. Schwenk, Phys. Rev. Lett. 122 (2019) 042501.

[119] F. Sammarruca, L.E. Marcucci, L. Coraggio, J.W. Holt, N. Itaco, and R. Machleidt, "Nuclear and Neutron Matter Equations of State from High-Quality Potentials up to Fifth Order of the Chiral Expansion", arXiv:1807.06640 [nucl-th].

[120] C. Drischler, J.A. Melendez, R.J. Furnstahl, and D.R. Phillips, Phys. Rev. C 102 (2020) 054315.

[121] P.F. Bedaque, H.-W. Hammer, and U. van Kolck, Phys. Rev. Lett. 82 (1999) 463.

[122] P.F. Bedaque, H.-W. Hammer, and U. van Kolck, Nucl. Phys. A 646 (1999) 444.

[123] P.F. Bedaque, H.-W. Hammer, and U. van Kolck, Nucl. Phys. A 676 (2000) 357.

[124] E. Braaten and H.-W. Hammer, Phys. Rept. 428 (2006) 259.

[125] L. Platter, H.-W. Hammer, and U.-G. Meißner, Phys. Rev. A 70 (2004) 052101.

[126] L. Platter, H.-W. Hammer, and U.-G. Meißner, Phys. Lett. B 607 (2005) 254.

[127] B. Bazak, J. Kirscher, S. König, M. Pavón Valderrama, N. Barnea, and U. van Kolck, Phys. Rev. Lett. 122 (2019) 143001. 
[128] S. König, H.W. Grießhammer, H.-W. Hammer, and U. van Kolck, J. Phys. G 43 (2016) 055106.

[129] S. König, H. W. Grießhammer, H.-W. Hammer, and U. van Kolck, Phys. Rev. Lett. 118 (2017) 202501.

[130] S. König, Eur. Phys. J. A 56 (2020) 113.

[131] A. Kievsky, M. Viviani, M. Gattobigio, and L. Girlanda, Phys. Rev. C 95 (2017) 024001.

[132] A. Kievsky, M. Viviani, D. Logoteta, I. Bombaci, and L. Girlanda, Phys. Rev. Lett. 121 (2018) 072701.

[133] Y.-H. Song, R. Lazauskas, and U. van Kolck, Phys. Rev. C 96 (2017) 024002 [erratum: Phys. Rev. C 100 (2019) 019901].

[134] C.-J. Yang, A. Ekström, C. Forssén, and G. Hagen, Phys. Rev. C 103 (2021) 054304.

[135] R. Machleidt, P. Liu, D.R. Entem, and E. Ruiz Arriola, Phys. Rev. C 81 (2010) 024001.

[136] C.-J. Yang, A. Ekström, C. Forssén, G. Hagen, G. Rupak, and U. van Kolck, in preparation.

[137] R. Machleidt, K. Holinde, and C. Elster, Phys. Rept. 149 (1987) 1.

[138] M. Pavón Valderrama and D.R. Phillips, Phys. Rev. Lett. 114 (2015) 082502.

[139] S. Weinberg, Phys. Rev. Lett. 43 (1979) 1566.

[140] V. Cirigliano, W. Dekens, J. de Vries, M.L. Graesser, E. Mereghetti, S. Pastore, and U. van Kolck, Phys. Rev. Lett. 120 (2018) 202001.

[141] V. Cirigliano, W. Dekens, J. de Vries, M.L. Graesser, E. Mereghetti, S. Pastore, M. Piarulli, U. van Kolck, and R. B. Wiringa, Phys. Rev. C 100 (2019) 055504.

[142] T.R. Richardson, M.R. Schindler, S. Pastore, and R.P. Springer, Phys. Rev. C 103 (2021) 055501.

[143] R. Wirth, J.M. Yao, and H. Hergert, "Ab Initio Calculation of the Contact Operator Contribution in the Standard Mechanism for Neutrinoless Double-Beta Decay", arXiv:2105.05415 [nucl-th].

[144] Z. Davoudi and S.V. Kadam, Phys. Rev. Lett. 126 (2021) 152003.

[145] E.E. Jenkins and A.V. Manohar, Phys. Lett. B 259 (1991) 353.

[146] M.N. Butler and M.J. Savage, Phys. Lett. B 294 (1992) 369. 
[147] T.R. Hemmert, B.R. Holstein, and J. Kambor, J. Phys. G 24 (1998) 1831.

[148] N. Fettes and U.-G. Meißner, Nucl. Phys. A 679 (2001) 629.

[149] V. Pascalutsa and D.R. Phillips, Phys. Rev. C 67 (2003) 055202.

[150] B. Long and U. van Kolck, Nucl. Phys. A 840 (2010) 39.

[151] S.R. Beane and U. van Kolck, J. Phys. G 31 (2005) 921.

[152] B. Long and U. van Kolck, Nucl. Phys. A 870-871 (2011) 72.

[153] J. Fujita and H. Miyazawa, Prog. Theor. Phys. 17 (1957) 360.

[154] V.R. Pandharipande, D.R. Phillips, and U. van Kolck, Phys. Rev. C 71 (2005) 064002.

[155] M. Piarulli, L. Girlanda, R. Schiavilla, A. Kievsky, A. Lovato, L.E. Marcucci, S.C. Pieper, M. Viviani, and R.B. Wiringa, Phys. Rev. C 94 (2016) 054007.

[156] M. Piarulli, A. Baroni, L. Girlanda, A. Kievsky, A. Lovato, E. Lusk, L.E. Marcucci, S.C. Pieper, R. Schiavilla, M. Viviani, and R.B. Wiringa, Phys. Rev. Lett. 120 (2018) 052503 .

[157] A. Ekström, G. Hagen, T.D. Morris, T. Papenbrock, and P. D. Schwartz, Phys. Rev. C 97 (2018) 024332.

[158] W.G. Jiang, A. Ekström, C. Forssén, G. Hagen, G. R. Jansen, and T. Papenbrock, Phys. Rev. C 102 (2020) 054301.

[159] I. Tews, Z. Davoudi, A. Ekström, J.D. Holt, and J.E. Lynn, J. Phys. G 47 (2020) 103001.

[160] S.R. Beane, P.F. Bedaque, L. Childress, A. Kryjevski, J. McGuire, and U. van Kolck, Phys. Rev. A 64 (2001) 042103.

[161] M. Pavón Valderrama and E. Ruiz Arriola, Annals Phys. 323 (2008) 1037.

[162] J. Schwinger, Phys. Rev. 61 (1942) 387.

[163] M. Rho, "The 'Folk Theorem' on Effective Field Theory: A case for nuclear physics", arXiv:2104.01899 [nucl-th].

[164] U. van Kolck, Front. in Phys. 8 (2020) 79.

[165] U. van Kolck, Eur. Phys. J. A 56 (2020) 97. 